\title{
Enhancement of coxsackievirus B3 infection by antibody to a different coxsackievirus strain
}

\author{
Jaskamal Girn, Mojgan Kavoosi and Janet Chantler \\ Department of Pathology and Laboratory Medicine, University of British Columbia, \#318, BCRICWH, 950 West 28th Avenue, Vancouver, \\ British Columbia, Canada V5Z 4H4
}

\begin{abstract}
Group B coxsackieviruses (CVBs) are a major cause of viral myocarditis and pancreatitis in humans and produce a similar pattern of disease in inbred strains of mice. As there are six strains of CVBs, individuals can be infected with multiple serotypes. This raises the possibility of antibody enhancement of infectivity (AEI) by cross-reactive but non-neutralizing antibody to a different strain from a prior infection. To determine whether AEI plays a role in coxsackievirus pathogenesis, an in vitro system using the murine macrophage cell line J774.1 was tested for enhanced infection when incubated with CVB3 plus anti-CVB2 antibody. Yields of virus were found to increase by 10-50-fold and the percentage of infected cells increased proportionately. The effect was Fcmediated as $\mathrm{F}\left(\mathrm{ab} \mathrm{b}^{\prime}\right)_{2}$ fragments of the antibody could not mediate the effect. To determine whether AEI could also be demonstrated in vivo CVB3 was injected into 5-week-old mice together with mouse polyclonal anti-CVB2. Controls included mice injected with PBS or CVB3 alone. Results showed that the titres of virus in tissues of animals injected with virus plus antibody were 1-2 logs higher than when virus was injected alone. This was accompanied by greater histopathological damage, particularly in the heart. These results have implications for human disease as infection with multiple strains likely occurs during the lifetime of an individual.
\end{abstract}

\section{Introduction}

The coxsackieviruses are members of the family Picornaviridae, genus Enterovirus and are divided into two groups depending on their pathogenesis in newborn mice. Group A coxsackieviruses infect muscle tissue, inducing a flaccid paralysis, while group $\mathrm{B}$ viruses (CVBs) have a much broader tissue tropism, infecting the CNS, exocrine pancreas, spleen, liver, brown fat and heart muscle. There are six serotypes of CVB, which cross-react serologically but have distinct neutralizing epitopes and therefore a single individual can be infected with multiple strains during his/her life. The association of CVBs with heart disease has been the focus of considerable attention as serological studies have indicated that these viruses are the most frequent cause of acute viral myocarditis (Grist \& Reid, 1994) and may also play a role in dilated cardiomyopathies in humans. Apart from the presence of elevated antibody titres, viral genome has also frequently been

Author for correspondence: Janet Chantler.

e-mail chantler@interchange.ubc.ca identified in biopsy specimens from patients with chronic myocardial disease by in situ hybridization (ISH) or PCR (Bowles et al., 1986; Archard et al., 1987; Easton \& Eglin, 1988).

Heart disease associated with CVB infection usually occurs in adulthood although serological studies indicate widespread exposure of the population to this group of viruses during childhood. Moreover, patients presenting with acute myocarditis have been reported to have antibody against a greater number of coxsackieviruses than age-matched controls, indicating that they are not undergoing a primary CVB infection at the time of development of clinical cardiomyopathy (Horr et al., 1981). The possibility that secondary infection with a different strain of CVB might have a role in pathogenesis was investigated by Beck et al. (1990) in a murine model of coxsackievirus myocarditis. These investigators showed that tandem infection of $\mathrm{C} 3 \mathrm{H} / \mathrm{HeJ}$ mice with $\mathrm{CVB} 2$ followed 1 month later by CVB3 resulted in more intense myocarditis than found in animals injected with either CVB2 or CVB3 alone. They concluded that the effect was due to cell-mediated immune responses to a conserved enterovirus epitope, al- 
though the mechanism of this was not further investigated. Another explanation for the phenomenon they observed is antibody enhancement of the secondary CVB3 infection by cross-reactive antibody from the antecedent inoculation with CVB2. Such exacerbation of virus infections by pre-existing antibody which reacts with virus particles but is nonneutralizing has been observed with a number of viruses, notably members of the Flaviviridae such as dengue (Halstead, 1982; Halstead \& O'Rourke, 1977) and yellow fever virus (Porterfield, 1986) as well as respiratory syncytial virus (Gimenez et al., 1996) and recently human immunodeficiency virus (Montefiori et al., 1996). Antibody enhancement of infectivity (AEI) of disease is believed to be due to uptake of virus-antibody complexes via Fc receptors on cells (usually macrophages) with subsequent replication and release of virus resulting in higher levels of viraemia and thus virus reaching the target tissues. AEI is believed to be responsible for the haemorrhagic fever accompanying secondary dengue virus infections (dengue shock syndrome) and has been suggested to have a role in AIDS pathogenesis (Homsy et al., 1989).

The study reported here examines the possible role of cross-reactive antibody in mediating the enhancement of myocarditis seen in secondary CVB infections in mice. The effect of polyclonal anti-CVB2 antibody (IgG fraction) on CVB3 infection of macrophages in vitro is reported and also the result of injecting susceptible strains of mice with CVB3 together with anti-CVB2 IgG.

\section{Methods}

Cell culture. Vero cells and J774.1 cells were obtained from the ATCC. Vero cells were maintained in DMEM-F12 medium supplemented with 10\% FBS and 1\% gentamycin. J774.1 cells were maintained in DMEM containing $2 \mathrm{mM}$ glutamine, 10\% heat-inactivated FBS and $1 \%$ gentamycin. Cells were grown at $37{ }^{\circ} \mathrm{C}$ in a humidified incubator in $5 \%$ $\mathrm{CO}_{2}$.

Virus. The RK (Reinhardt Kandolf) strain of coxsackievirus B3 was derived from an infectious clone, $\mathrm{pCVB} 3 / \mathrm{T} 7$, that was transcribed with T7 polymerase and the RNA subsequently transfected into HeLa cells. The progeny virus was passaged in mice and the virus obtained from heart tissue is the strain that we received from R. Kandolf's laboratory and designated CVB3(RK). Stock virus was prepared by infection of Vero cells at 0.1 p.f.u. per cell. When $100 \%$ cytopathology was evident at 24-48 h post-infection, the cultures were freeze-thawed twice to release intracellular virus into the medium which was then centrifuged at $800 \mathrm{~g}$ for $10 \mathrm{~min}$ at $4{ }^{\circ} \mathrm{C}$ to remove cell debris. The supernatant was stored in aliquots at $-70^{\circ} \mathrm{C}$, and was titred in Vero cells by plaque assay.

Production of anti-CVB2 antibody. Polyclonal anti-CVB2 IgG was prepared in BALB/c mice against the Ohio strain of CVB2, obtained from the ATCC. The mice were injected intra-peritoneally with $10^{5}$ p.f.u. of CVB2 and were sacrificed after 4 weeks. Approximately $0.8 \mathrm{ml}$ of intraventricular blood was obtained from each mouse and allowed to clot at $4{ }^{\circ} \mathrm{C}$. The serum obtained was pooled prior to immunoglobulin purification. This was carried out by ammonium sulphate precipitation and fractionation by protein A-Sepharose chromatography.

Virus infection of J774.1 cells and peritoneal macro- phages. Cells were infected at 5-20 p.f.u. per cell for $1 \mathrm{~h}$. After removal of the inoculum the cells were washed three times and then incubated in growth medium containing $5 \%$ heat-inactivated FBS and $1 \%$ gentamycin. At $2 \mathrm{~h}$ intervals the cultures were harvested and freeze-thawed twice to release virus from the cells. Virus was titred by plaque assay.

Infectious focus assay. Cells were infected and incubated as before. At $2 \mathrm{~h}$ intervals, cultures were harvested, the cells were washed three times in DMEM and then plated at known numbers in $35 \mathrm{~mm}$ dishes containing a monolayer of Vero cells. After gently centrifuging the cells onto the monolayer, the supernatant was removed and the cells were overlaid with agar. Plaques were stained and counted after 2 days, and the numbers were correlated with the number of cells plated.

Infection of mice. Male A/J mice (4-5 weeks old) were obtained from Jackson Laboratories. The animals were allowed to acclimatize for 1 week before inoculation with $0 \cdot 2 \mathrm{ml}$ PBS containing $10^{4}$ p.f.u. of CVB3 alone or together with $10 \mu \mathrm{l}$ of anti-CVB2 antibody. On days 3 or 5 postinfection five animals of each group were anaesthetized with sodium pentobarbital ( $60 \mathrm{mg}$ per $\mathrm{kg}$ ) and heart, spleen, kidney, pancreas, liver and lung were removed aseptically. The tissues were sectioned transversely into two pieces, one section was fixed with $4 \%$ paraformaldehyde solution for histopathology and in situ hybridization and the other was snap-frozen in liquid nitrogen for plaque titration.

Plaque titration of virus in organs. Snap-frozen sections were homogenized in $1 \mathrm{ml}$ of DMEM and the homogenate was then centrifuged to remove the cell debris at $12000 \mathrm{~g}$. The supernatant was stored at $-70{ }^{\circ} \mathrm{C}$ for plaque assay.

Histology. Sections ( $3 \mu \mathrm{m}$ thick) were prepared from organs fixed in $4 \%$ paraformaldehyde solution using a Leica microtome. The sections were stained with haemotoxylin and eosin and Masson's trichrome to assess histopathological damage.

In situ hybridization. Tissues were fixed with $4 \%$ paraformaldehyde solution overnight, rinsed with PBS, embedded in paraffin blocks, cut into $4 \mu \mathrm{m}$ sections and placed on silanated glass slides. The sections were baked overnight at $60^{\circ} \mathrm{C}$, deparaffinized using xylene and rehydrated in graded alcohols. The tissues were permeabilized using $0.2 \mathrm{M} \mathrm{HCl}, 2 \times \mathrm{SSC}, 20 \mathrm{mM}$ Tris $/ 2 \mathrm{mM} \mathrm{CaCl}_{2}$ containing $1 \mu \mathrm{g} / \mathrm{ml}$ of proteinase $\mathrm{K}$, and $0.25 \%$ acetic anhydride containing $0.1 \mathrm{M}$ triethanolamine. The slides were then dehydrated using graded alcohols. Once the tissues were completely dried, $25 \mu \mathrm{l}$ of the hybridization solution containing $100 \mathrm{ng} / \mathrm{ml}$ of the DIG-labelled sense or anti-sense probe was added to each section. The sections were then covered with glass coverslips and placed in a sealed humidified dish at $42{ }^{\circ} \mathrm{C}$ overnight. Post-hybridization washes were performed overnight using $50 \%$ formamide, $10 \mathrm{mM}$ Tris/ImM EDTA and $600 \mathrm{mM} \mathrm{NaCl}$ in a $56{ }^{\circ} \mathrm{C}$ rocking water bath, followed by several washes in $2 \times$ SSC. The slides were equilibrated in buffer 1 containing $0.15 \mathrm{M} \mathrm{NaCl}$ and $0.1 \mathrm{M}$ Tris- $\mathrm{HCl}$ and blocked with $2 \%$ lamb serum. Anti-DIG-alkaline phosphatase (100 $\mu \mathrm{l}$; diluted 1:500 in buffer 1 containing $1 \%$ lamb serum and $0.1 \%$ Tween-20) was added per section and incubated for $45 \mathrm{~min}$ at room temperature in a humidified chamber. The slides were washed with buffer $\mathrm{I}$ and were equilibrated to $\mathrm{pH} 9 \cdot 5$ in buffer 3 . The alkaline phosphataselinked anti-DIG antibody was detected by incubation with NBT/BCIP substrate (Sigma fast tablets) for $24 \mathrm{~h}$ at room temperature. The slides were counterstained with eosin and were examined for a positive reaction indicated by a blue-black colour.

The ISH score is an arbitrary scale of $0-5$, where 0 represents no viral genome detected and 5 represents massive detection of bound probe at high levels over $25-30 \%$ of the heart section. It is used to record a 
composite value for the amount of viral genome detected in all the mice in one set.

\section{Results}

\section{Susceptibility of murine macrophages to CVB3}

The underlying requirement for AEI is that the Fc bearing cell is at least partially restrictive to virus uptake but permissive to virus that gains entry to the cytosol. To determine the degree of susceptibility of murine macrophages to CVB3, virus growth curves were determined in both freshly isolated peritoneal macrophages and the macrophage cell line J774.1. Cells were infected at 5-20 p.f.u. per cell and following removal of the inoculum, amounts of progeny virus were determined over $24 \mathrm{~h}$. The results are shown in Fig. 1 in comparison with virus growth in Vero cells, which are highly permissive to virus growth.

In Vero cells, virus progeny can be detected by $4 \mathrm{~h}$ postinfection and reach a peak at $8 \mathrm{~h}$ when titres of virus are approximately $10^{6}$ p.f.u. $/ \mathrm{ml}$. By $24 \mathrm{~h}$ post-infection, no new virus is being produced and massive cytopathology is apparent with the majority of cells rounded and floating. In the J774.I cells the time-course of virus release is similar but the titres of virus obtained are 100-fold lower. Moreover, although new virus production has ceased by $24 \mathrm{~h}$, this is not accompanied by cell death but apparently represents clearing of the infection, leaving an intact monolayer of J774.1 cells which can be cultured indefinitely. The cultures of peritoneal macrophages were even more restrictive to virus replication with yields of only $5 \times 10^{2}-10^{3}$ p.f.u. over a background of approximately $10^{2}$ p.f.u. that remained after adsorption of the inoculum.

\section{Determination of the percentage of infected cells in each culture}

Infectious focus assays were performed to determine the proportion of infected cells in each of the cultures. The results showed that at $6 \mathrm{~h}$ post-infection, essentially all ( $>95 \%$ ) Vero cells were infected when high multiplicities of infection (5-20 p.f.u. per cell) were used. In contrast only 3-6\% of J774.I cells were infected and an even smaller proportion of peritoneal macrophages $(0.5-1 \cdot 5 \%)$, explaining at least in part the low yields of virus with these cells.

\section{Effect of anti-CVB2 antibody on CVB3 infection of J774. 1 cells}

Aliquots of CVB3 virus $\left(2 \times 10^{7}\right.$ p.f.u. $)$ were mixed with $1: 500$ and $1: 1000$ dilutions of anti-CVB2 $\mathrm{IgG}$, or with an equivalent volume of PBS, and were incubated with $1 \times 10^{6}$ J774. I cells for $1 \mathrm{~h}$. The inoculum was then removed and the cells were washed three times with PBS and then incubated in RPMI medium. Supernatant medium was harvested and replaced at 2, 4, 6, 8 and $24 \mathrm{~h}$ post-infection and was titred on Vero cells. The result is shown in Fig. 2.

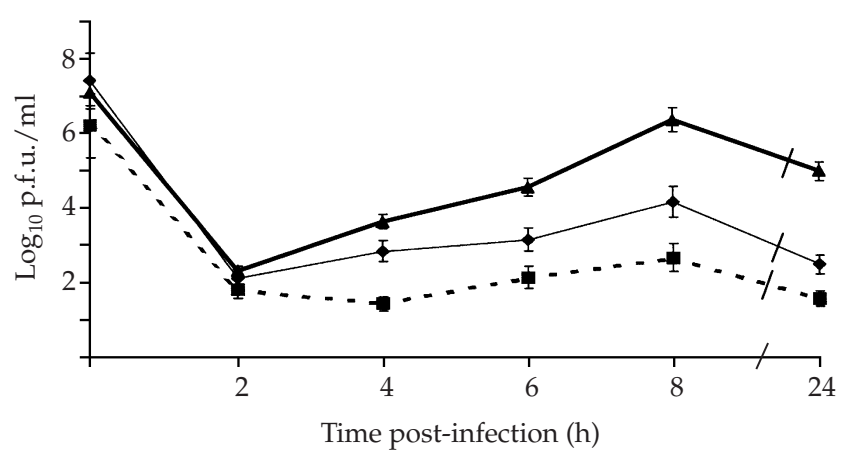

Fig. 1. Time-course of production of progeny CVB3 virus in $\mathbf{3 7 7 4 . 1}$ cells $(\bullet)$, peritoneal macrophages $(\boldsymbol{\square})$ and Vero cells $(\boldsymbol{\Delta})$. The $t=0$ timepoint represents input virus and titres are expressed as $\log _{10}$ p.f.u. $/ \mathrm{ml}$.

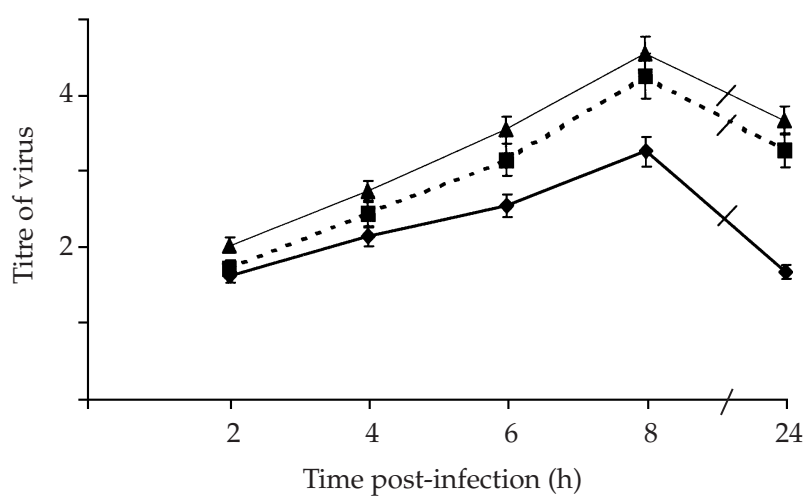

Fig. 2. The effect of anti-CVB2 antibody on CVB3 replication in 5774.1 cells. The cells were infected at 5 p.f.u. per cell together with a 1:500 ( $\mathbf{a})$ or 1:1000 ( $\mathbf{A})$ dilution of polyclonal anti-CVB2 antibody or with CVB3 alone $(\boldsymbol{)})$. Titres of virus at $2,4,6,8$ and $24 \mathrm{~h}$ were determined.

CVB3 in the absence of anti-CVB2 showed a similar growth curve in J774.I cells to that seen in Fig. I, with yields at $8 \mathrm{~h}$ of $1.3 \times 10^{3}$ p.f.u. $/ \mathrm{ml}$. In contrast the amounts of released virus in the cultures infected with CVB3 plus antiCVB2 antibody (at either dilution) were 50-100-fold higher at $8 \mathrm{~h}$ post-infection.

Infectious focus assays were also performed on J774.1 cells infected in the presence and absence of different dilutions of anti-CVB2 IgG and harvested at $6 \mathrm{~h}$ post-infection. The results of two separate experiments are shown in Fig. 3. The proportion of infected cells increased 10-20-fold in the presence of $1: 500$ or $1: 1000$ dilutions of anti-CVB2 but the enhancement was lost with the 1:5000 dilution of antibody (Fig. 3).

\section{Enhancement of CVB3 infection by anti-CVB2 $F\left(a b^{\prime}\right)_{2}$ fragments}

Enhancement of virus infection by antibody is believed to work by attachment of infectious virus-antibody complexes to cells via Fc receptors. Removal of the Fc portion of the antibody should therefore abrogate this process while still 


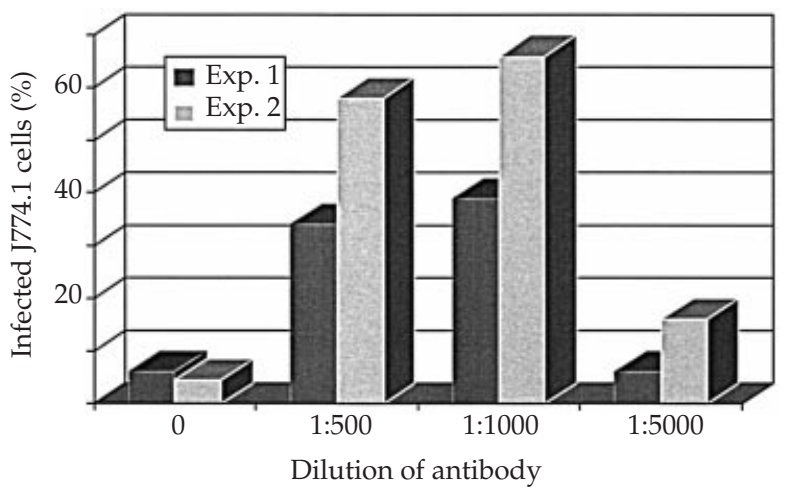

Fig. 3. The percentage of J774.1 cells infected in the presence and absence of anti-CVB2 antibody was determined by infectious focus assay. The results of two representative experiments are shown.

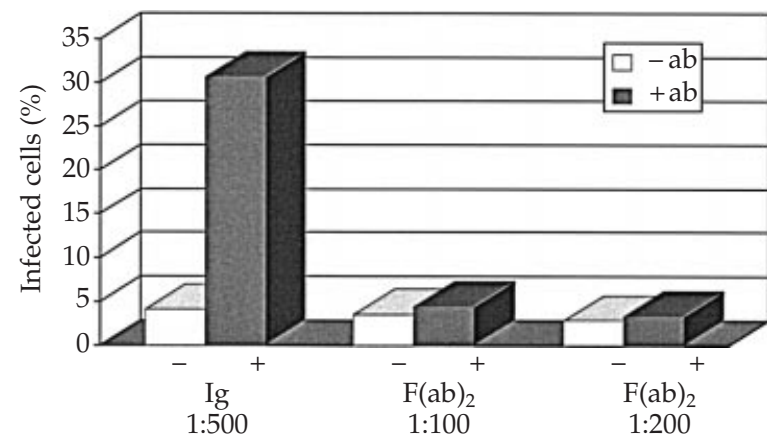

Fig. 4. The ability of an $F\left(a b^{\prime}\right)_{2}$ fragment of anti-CVB2 antibody to enhance uptake of CVB3 into J774.1 cells was determined. The percentage of infected cells in the presence or absence of $1: 500$ dilution of whole immunoglobulin is compared with $1: 100$ and $1: 200$ dilutions of $\left.\mathrm{F}(\mathrm{ab})_{2}\right)_{2}$ fragment.

allowing the $\mathrm{F}\left(\mathrm{ab} \mathrm{b}_{2}\right)_{2}$ fragment to attach to the virus particle. To determine whether the enhancement seen was Fc-mediated, anti-CVB2 IgG was treated with pepsin and undigested antibody was removed by protein A-Sepharose chromatography. The $\mathrm{F}\left(\mathrm{ab} \mathrm{b}_{2}\right)_{2}$ fragment (at a 1:10 dilution) was shown to have an ELISA reading comparable to a 1:50 dilution of the complete IgG molecule (absorbance at $405 \mathrm{~nm}$ of 0.50 and 0.54 respectively).

Using equivalent amounts of $F\left(a b^{\prime}\right)_{2}$ to original $\mathrm{IgG}$, the previous experiment to assess the proportion of infected J774.I cells was repeated and the result is shown in Fig. 4.

As expected, no enhancement was seen with the $\mathrm{F}\left(\mathrm{ab}^{\prime}\right)_{2^{\prime}}$ while a 10-fold increase was seen with a 1:500 dilution of the undigested $\operatorname{IgG}$ molecule.

\section{Effect of anti-CVB2 IgG on the pathogenesis of CVB3 infection in $\mathrm{A} / \mathrm{J}$ mice}

Groups of five mice (5 weeks old) were injected intraperitoneally with $10^{5}$ p.f.u. of CVB3 alone or together with

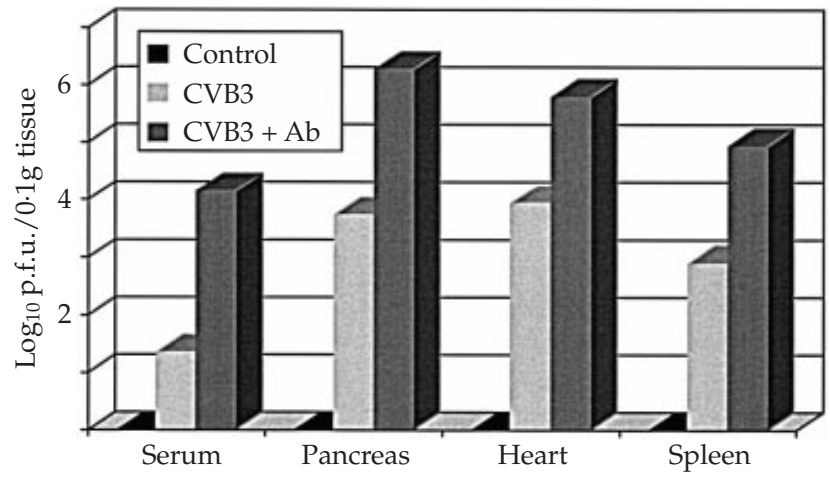

Fig. 5. A/J mice were inoculated intraperitoneally with $10^{4}$ p.f.u. CVB3 alone or with anti-CVB2 antibody. Animals were sacrificed on day 5 and tissues were frozen for plaque titration. Results are expressed as $\log _{10}$ p.f.u. $/ \mathrm{ml}$ of serum or per $0.1 \mathrm{~g}$ of tissue.

anti-CVB2 IgG. Control mice received an equivalent volume of PBS. The animals were sacrificed on days 3 or 5 post-injection and samples of serum, pancreas, heart and spleen were removed for plaque assay, histopathology and in situ hybridization.

\section{Virus titres}

The levels of infectious virus detected in the tissues are shown in Fig. 5. In serum, 1000-fold higher titres were detected in animals injected with CVB3 plus anti-CVB2 than in those injected with CVB3 alone. Similarly, much higher levels of virus (about 100-fold) were found in pancreas, heart and spleen on day 5 post-inoculation.

\section{Histopathology and in situ hybridization (ISH)}

Sections of tissue were treated with Masson's trichrome stain for assessment of tissue damage and ISH was performed to determine the amount of viral genome present.

Pancreas. Massive necrosis of exocrine pancreas can be seen in Fig. 6(c, e, g) in animals injected with virus alone, or with virus plus antibody, in comparison with the control tissue in Fig. 6(a). Interestingly, CVB3 does not infect pancreatic islet tissue and intact islets can be seen, despite the widespread destruction of the surrounding tissue.

The amount of CVB3 genome detected in pancreas is shown in Fig. 6(d, f, h). By day 5 post-infection, the virus has been largely eliminated by the immune system from pancreas, which shows maximal levels of infection on day 3 (Fig. 6d). However, the mice injected with virus plus antibody showed higher levels of residual genome (ISH score of 2.5) than those infected with virus alone (ISH score of 0.5). This corresponds with the higher virus titres seen in these animals.

Heart. The degree of damage to heart tissue in animals injected with CVB3 in the presence or absence of anti-CVB2 IgG is 

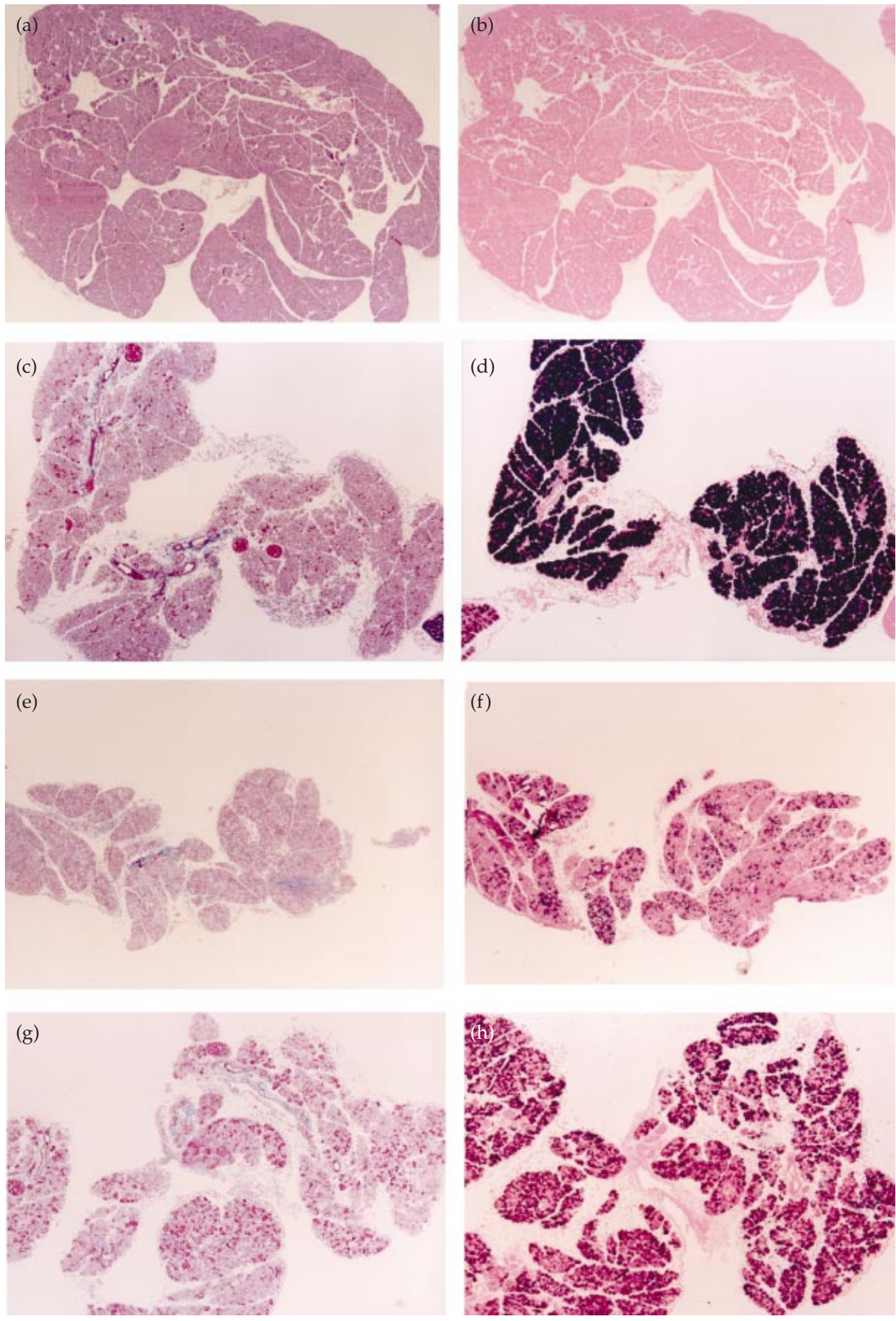

Fig. 6. The degree of tissue damage and the amount of viral genome present in pancreatic tissue of $A / J$ mice infected with CVB3 alone or together with anti-CVB2 antibody is shown. ( $a, b)$ Control pancreas; (c, d) CVB3-infected, day 3 postinfection; (e, f) CVB3-infected, day 5 post-infection; ( $g, h$ ) CVB3-infected + anti-CVB2, day 5 post-infection. (a, c, e, g) Masson's trichrome; (b, d, f, h) in situ hybridization.

shown in photomicrographs of transverse heart sections in Fig. 7. Tissue pathology seen in animals injected with CVB3 alone, on day 5 post-infection, was assessed as minor, while widespread necrosis was apparent in animals injected with virus plus antibody. A similar result was seen by in situ hybridization (Fig. 8), where the size of lesions containing CVB3 genome and the amount of genome detected can be seen to be much greater in mice injected with virus plus 

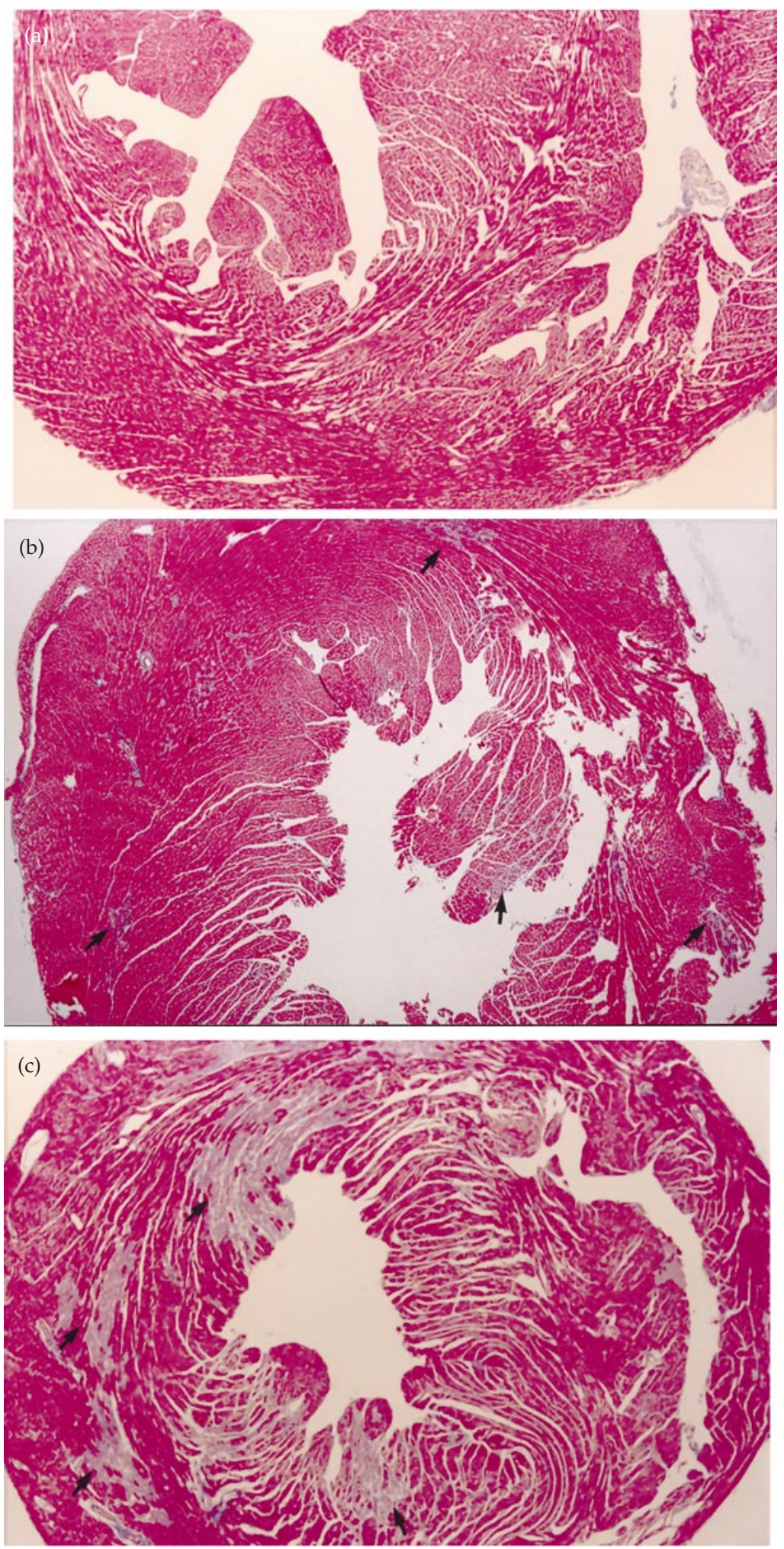

Fig. 7. Heart damage in A/J mice inoculated with CVB3 alone or together with anti-CVB2 antibody (Masson's trichrome stain). (a) Control; (b) CVB3-infected; (c) CVB3-infected + anti-CVB2 antibody. 

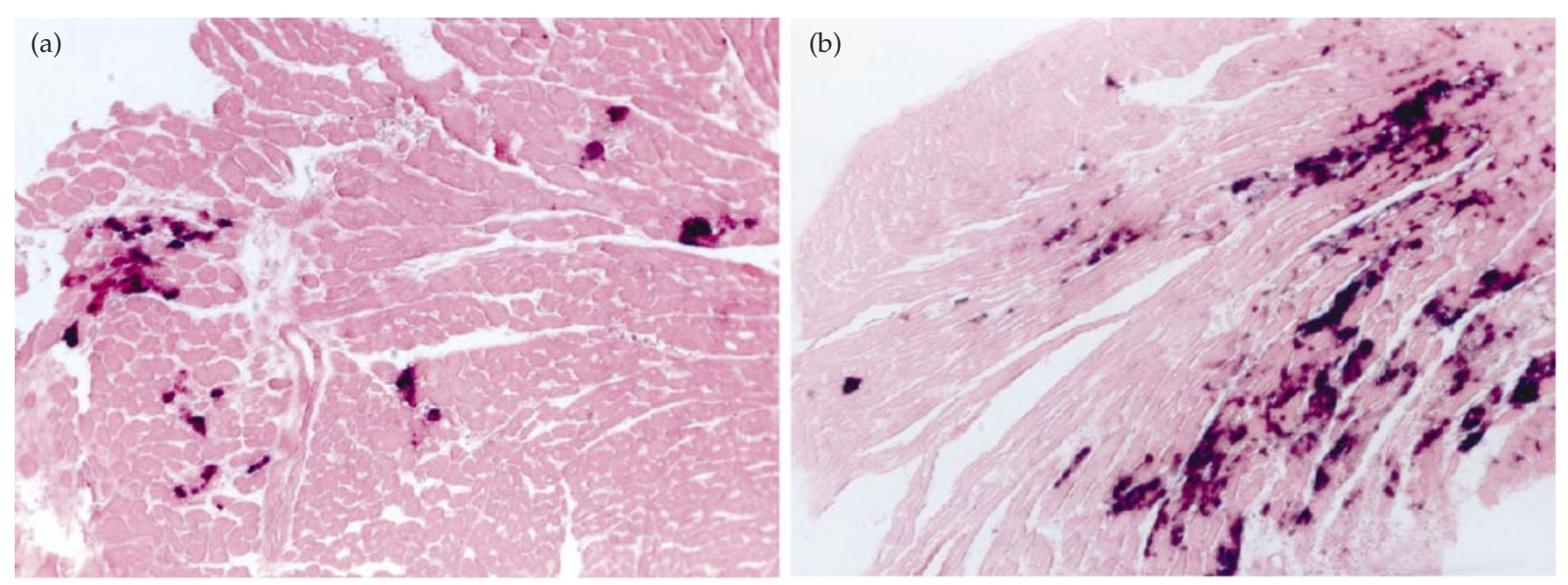

Fig. 8. In situ hybridization to assess CVB3 viral genome in heart tissue of A/J mice on day 5 post-infection. (a) CVB3 alone; (b) CVB3 + anti-CVB2 antibody.

antibody (ISH score of 3.5) than in mice injected with virus alone (ISH score of 1 ).

\section{Discussion}

The results presented clearly show that CVB3 infection can be enhanced by antibody to a different coxsackievirus strain which is cross-reactive but non-neutralizing. The effect has been shown in cultured macrophages (J774.1 cells) and is Fcmediated as pepsin-digested IgG was no longer able to mediate the effect. The mechanism is therefore believed to involve the uptake of infectious virus-antibody complexes into Fc-receptor-bearing cells, although the possibility that the antibody merely stabilizes virus particles has not been eliminated. Once having gained entry to the cell, the virus replicates, producing high levels of progeny. AEI occurs with CVB3 as the virus is normally highly restricted in uptake into macrophage-like cells, as shown by the infectious focus assay which determined that only 3-6\% of J774.1 cells and $1 \%$ of peritoneal macrophages are normally infected. However these cells are permissive to virus that gains entry to the cytoplasm, as indicated by the approximately 100 -fold increase in virus yields seen in the J774.I cultures where antibody was present.

AEI was also demonstrated in vivo when CVB3 was injected together with anti-CVB2 IgG into 5-week-old A/J mice (intra-peritoneally). Animals injected with virus plus antibody showed 1000-fold increases in circulating virus and 50-100-fold increases in virus titre in several organs including pancreas and heart. We interpret this as follows. In animals with pre-existing antibody to a different CVB strain, virus titres may be augmented by enhanced replication in local macrophages at the site of entry. In the case of the enteroviruses, this would occur in Peyer's patches in the mucosal layer of the small intestine and in nearby mesenteric lymph nodes (Melnick, 1996). This in turn would raise the level of viraemia and therefore the amount of virus reaching the target tissues. In tissues that are susceptible to CVB3, the larger infectious load would cause more cells to be infected and therefore increase the amount of tissue pathology induced before the immune system was able to bring the infection under control.

These findings have considerable implications for understanding CVB pathogenesis and are in agreement with the results of Beck et al. (1990) who showed that tandem infections of mice with CVB2 followed I month later by CVB3 also resulted in much more serious disease. While their interpretation of the results related to T-cell reactivity to a conserved CVB epitope, it would seem likely from our study that enhancement by antibody was at least partly the explanation. The relevance of these results to humans relates to the fact that there are six strains of CVB and the majority of individuals become infected by multiple strains during their lifetime. The possibility therefore exists that a CVB infection may be exacerbated by pre-existing antibody to a different strain, resulting in greater tissue damage in heart and other organs. This correlates with the known increase in acute viral myocarditis with age (McManus et al., 1986) and exposure to multiple CVB strains (Horr et al., 1981).

This project was supported by a grant from the Heart and Stroke Foundation of Canada.

\section{References}

Archard, L. C., Bowles, N. E., Olsen, E. G. J. \& Richardson, P. J. (1987). Detection of persistent coxsackie B virus RNA in dilated cardiomyopathies and myocarditis. European Heart Journal 8 (Suppl. J), 437-440.

Beck, M. A., Tracy, S., Mullican, C. \& McManus, B. M. (1990). Secondary enterovirus infection in the murine model of viral infectivity. American Journal of Pathology 136, 669-680.

Bowles, N. E., Richardson, P. J. \& Olsen, E. G. J. (1986). Detection of coxsackie-B-virus-specific RNA in myocardial samples. Lancet i, 11201123. 
Easton, A. J. \& Eglin, R. P. (1988). The detection of coxsackievirus RNA in cardiac tissue by in situ hybridization. Journal of General Virology 69, 285-291.

Gimenez, H. B., Chisholm, S., Dornan, J. \& Cash, P. (1996). Neutralizing and enhancing activities of human respiratory syncytial virus-specific antibodies. Clinical and Diagnostic Laboratory Immunology 3 , 280-286.

Grist, N. R. \& Reid, D. (1994). Epidemiology of viral infections of the heart. In Viral Infections of the Heart, chapter 2, pp. 23-30. Edited by J. E. Banatvala. Oxford: Oxford University Press.

Halstead, S. B. (1982). Immune enhancement of viral infectivity. Progress in Allergy 31, 301-364.

Halstead, S. B. \& O'Rourke, E. J. (1977). Antibody-enhanced dengue virus infection in primate leukocytes. Nature 265, 739-741.

Homsy, J., Meyer, M., Tateno, M., Clarkson, S. \& Levy, J. A. (1989). The FC and not CD4 receptor mediates enhancement of HIV infection in human cells. Science 244, 1357-1360.

Horr, H., Matoba, T., Shingu, M. \& Toshima, H. (1981). The role of cell- mediated immunity in coxsackie B viral myocarditis. Japanese Circulation Journal 45, 1409-1414.

McManus, B., Gauntt, C. \& Cassling, R. (1986). Immunopathological basis of myocardial injury. In Cardiovascular Clinics - Contemporary Issues in Cardiovascular Pathology, pp. 163-184. Edited by A. Brest. Philadelphia: Davis Publishing.

Melnick, J. L. (1996). Current status of poliovirus infection. Clinical Microbiology Reviews 9, 293-300.

Montefiori, D. C., Pantaleo, G., Fink, L. M., Zhou, J. T., Zhou, J. Y., Bilska, M., Miralles, G. D. \& Fauci, A. S. (1996). Neutralizing and infection-enhancing antibody responses to human immunodeficiency virus type 1 in long-term nonprogressors. Journal of Infectious Diseases 173, 60-67.

Porterfield, J. S. (1986). Antibody-dependent enhancement of viral infectivity. Advances in Viral Research 31, 335-355.

Received 27 October 2000; Accepted 25 September 2001 